\title{
Endoscopic Laser Treatment of Mucous Gland Adenoma Arising in the Trachea
}

I read with interest the case report in this issue by Ishida and colleagues (1) on a case of mucous gland adenoma of the trachea resected with Nd-YAG laser through a bronchoscope. Tracheal tumors are rare; there is one tracheal tumor to 180 lung tumors. In adults, tracheal tumors are most often malignant. Among benign tumors arising in the trachea, mucous gland adenoma is extremely rare. England and Hochholzer described that this tumor is generally solitary and arises in the lobar or segmental bronchus (2). Reports on mucous gland adenoma of the trachea are limited; the first report was described by Ferguson in 1988 (3).

See also p 890.

The serious complications related to laser therapy of the airway tumor are hemorrhage, respiratory distress and lung edema; hemorrhage may be the most common complication (4, 5 ). In the case reported in this issue, a cuffed endotracheal tube was inserted after local anesthesia to control the hemorrhage from the tracheal tumor related to the laser resection. The same device has been already employed by Shankar and colleagues in 1990 (6). However, it is more important to estimate the degree of hemorrhage before tumor resection or biopsy. In the case of polypoid lesion covered with intact airway epithelium, it is not easy to estimate the degree of hemorrhage as a serious complication of tumor resection based on macroscopical findings under bronchoscope. The authors tried to puncture the tracheal tumor with a fine needle through bronchoscope to estimate possible hemorrhage before the resection. The patient did not have massive hemorrhage by the needle puncture procedure and thus the tumor resection with endoscopic laser could be selected. This estimation using a needle is new technique and it may be useful to evaluate the degree of hemorrhage related to endoscopical tumor resection or biopsy.
Recent reports suggest that endoscopic laser therapy is curative in the case of benign tumor $(7,8)$. However, it is essential to make the correct diagnosis pathologically and to resect the tumor completely. In view of this point, device of Ishida et al (1) may be epochal to obtain enough material for pathological diagnosis and to resect the tumor completely without serious hemorrhage. Finally it is desired that this case will be followed up closely and the recurrence of tumor will be evaluated over for a long period, because cases of mucous gland adenoma are very rare and it may be potentially malignant.

\section{Takashi Dambara, MD, PhD, F.C.C.P. Department of Respiratory Medicine, Juntendo University School of Medicine, 2-1-1 Hongo, Bunkyo-ku, Tokyo 113}

\section{References}

1) Ishida $T$, Kamachi M, Hanada $T$, et al. Mucous gland adenoma of the trachea resected with an endoscopic Nd-YAG laser. Intern Med 35: 890, 1996.

2) England DM, Hochholzer L. Truly Benign "Bronchial Adenoma" Report of 10 Cases of Mucous Gland Adenoma with Immunohistochemical and Ultrastructural Findings. Am J Surg Pathol 19(8): 887, 1995.

3) Fergauson CJ, Cleeland JA. Mucus gland adenoma of the trachea: Case report and literature review. J Thorac Cardiovasc Surg 95(2): 347, 1988.

4) Tamura K, Hitomi S. Complications of Nd-YAG laser treatment on tracheobronchial lesions. Kyobu-Geka 45(6): 480, 1992.

5) Miro AM, Shivaram U, Finch PJ. Noncardiogenic pulmonary edema following laser therapy of a tracheal neoplasm. Chest 96(6): 1430, 1989.

6) Shankar S, George PJ, Hetzel MR, Goldstraw P. Elective resection of tumours of the trachea and main carina after endoscopic laser therapy. Thorax 45(6): 493, 1990.

7) Cavaliere S, Foccoli P, Farina PL. Nd:YAG laser bronchoscopy. A fiveyear experience with 1,396 applications in 1,000 patients. Chest 94(4): 959,1989

8) Kaiser LR. Management of tracheobronchial lesions with the Nd-YAG laser. Surg Clin North Am 67(5): 1087, 1987. 